\title{
Open-source modeling chain for the dynamic assessment of road traffic noise exposure
}

\author{
Valentin Le Bescond ${ }^{\mathrm{a}, \mathrm{c}}$, Arnaud Can ${ }^{\mathrm{a}, \mathrm{c}}$, Pierre Aumond ${ }^{\mathrm{a}, \mathrm{c}}$, Pascal Gastineau ${ }^{\mathrm{b}, \mathrm{c}}$ \\ a UMRAE, Univ Gustave Eiffel, IFSTTAR, CEREMA, F-44344 Bouguenais, France \\ b AME-SPLOTT, Univ Gustave Eiffel, IFSTTAR, F-44344 Bouguenais, France \\ c Institut de Recherche des Sciences et Techniques de la Ville (IRSTV), CNRS, 44321 Nantes Cedex 3, France
}

\begin{abstract}
In light of the growing concern about the adverse effects of noise pollution on health, a better understanding is needed of the relationships between urban transport and individual exposure. To improve the scientific community's modeling capabilities specific to this issue, we are proposing a noise exposure modeling framework that uses agent-based activity, multi-agent travel simulation and a European standardized noise emission and propagation model. Based on two open source software packages, MATSim and NoiseModelling, this framework aims to simulate the spatiotemporal distributions of daily individual activity and road traffic noise. The proposed approach makes it possible to use all the tools and methods proposed in the NoiseModelling software by importing MATSim outputs, therefore, taking full advantage of the development work carried out within the two communities. As such, it enables both characterizing the individual exposure to road traffic-related noise and investigating noise exposure inequality problems based on the attributes of individuals and their activities.
\end{abstract}

Keywords: noise exposure, activity-based model, multi-agent simulation, MATSim, NoiseModelling

\section{Introduction}


The significant health impacts from noise are universally acknowledged, with road traffic being rated as the primary contribution. Exposure-response relationships have been proposed, and the corresponding burden of disease in terms of disability-adjusted lifeyears has been quantified, in indicating that at least one million healthy life years are lost every year due to traffic-related noise in western Europe (WHO, 2011). Consequently, the World Health Organization strongly recommends reducing noise levels produced by road traffic to below 53 decibels $L_{\text {den }}$ and $45 \mathrm{~dB} L_{\text {night }}$ given that road traffic above these levels is associated with adverse effects on health and on sleep, respectively (WHO, 2018). Recent studies suggest that these recommendations may even be lowered (Vienneau et al., 2019; Van Kamp et al., 2020). Since they concentrate both the highest noise levels and population densities, metropolitan areas are subject to the highest exposures. Quantifying and reducing noise pollution exposure is thus a crucial issue for cities in terms of health, quality of life and attractiveness. In order to move towards cities in which all socioeconomic groups benefit from a quieter environment, it is necessary to improve understanding of the complex interactions taking place between transport policies and impacts in terms of distribution of noise exposure among the population. In this paper, we propose an open source modeling framework that enables both characterizing the individual exposure to road traffic-related noise and investigating noise exposure inequality problems.

The stakes involved in noise reduction have been assessed through both economic calculations correlating noise levels with property prices (Andersson et al., 2010) and contingent evaluation methods that estimate people's willingness to pay for a quieter environment (Lera-López, 2013; Barreiro, 2005). The correlation between noise exposure levels and socioeconomic groups has also been the subject of analyses; these studies most often conclude that disadvantaged populations are exposed to higher levels in their residential environment than affluent populations (Braubach and Fairburn, 2010). However, a recent study has shown that in Paris, France (excluding the suburbs), residents of more 
affluent neighborhoods were actually exposed to higher levels of road traffic noise compared to their less privileged counterparts (Havard et al., 2011). Although instructive, these quantified effects and environmental justice analyses are limited in their scope by virtue of being based on estimates of exposure levels in the place of residence. The actual exposure of individuals lies at an intersection between a field of noise pollution and individuals, both of which vary in time and space. In relying on concomitant noise and time-activity pattern measurements, Chaix (2018) and El Aarbaoui et al. (2017) demonstrated the strong influence of mobility on noise exposure.

From a decision-making perspective, traffic models are needed to assess the impact of mobility management policies on noise exposure. Some couplings between microscopic traffic models and noise prediction tools are already operational. These couplings make use of various traffic (SYMUVIA in Can et al. (2010), Chevallier et al. (2009) and Can et al. (2019); PARAMICS in De Coensel et al. (2010), De Coensel et al. (2012) and Luo et al. (2012); VISSIM in Estevez-Mauriz and Forssen (2019); HUTSIM in Heltimo et al. (2003)) and acoustic models (NMPB in Can et al. (2010); FHWA in Chevallier et al. (2019); HARMONOISE in De Coensel et al. (2010); IMAGINE in De Coensel et al. (2012); CNOSSOS in EstevezMauriz \& Forssen (2019) and Can et al. (2019); NORD in Heltimo et al. (2003)). The underlying principle is that the traffic model captures the effects of a traffic control strategy on vehicle kinematics, while the acoustic model calculates the resulting impact on vehicle emissions and moreover the levels in a set of receivers. Tested traffic control strategies include: a comparison between roundabouts and traffic signal intersections (Can et al., 2010; Chevallier et al., 2009; Estevez-Mauriz and Forssen, 2019), speed reduction measures (Luo et al., 2012), introduction of a dedicated bus lane (Can et al., 2010), green wave implementation (Can et al., 2010; De Coensel et al., 2010), traffic signal optimization (De Coensel et al., 2012), and demand management during morning rush hour (Can et al., 2019). Although various models have been used, all studies converge towards the expected result that reduced speeds and flows, combined with smoother traffic, serve 
to lower noise levels. However, with the exception of Can et al. (2019), which deals with a network spanning several $\mathrm{km}^{2}$, these studies are limited to very small areas and do not consider the reassignment of vehicles onto the network due to the tested strategy. In addition, all these studies ignore both multimodal trips and time-activity patterns in their exposure calculations.

In order to refine noise exposure calculations and assess the impact of transport policies on exposure, the development of efficient city-scale modeling chains, including a traffic module and an acoustic module, has thus become a key issue for the scientific community. Efficient city-scale acoustic models have been developed for noise mapping purposes. As regards traffic modeling, multi-agent traffic models are able to meet these requirements. Each agent in the simulation is an inhabitant whose trips are defined by a daily activity schedule and constrained by network traffic conditions. In addition, each agent is described by its socioeconomic attributes, hence paving the way for an assessment of environmental inequalities. Numerous studies have been carried out combining traffic agent-based models and air pollution models (see Ramacher and Karl (2020) for a review), in emphasizing the need to account for population dynamics when estimating population exposure. These models have been applied to reveal the relationships between exposure levels and socioeconomic groups. Among these studies, Gurram et al. (2019) showed that people living in poverty travel longer distances, tend to be of working age or are a minority group facing disproportionately high exposure levels. Moreover, Lu et al. (2019) found that exposure to $\mathrm{NO}_{2}$ is greater among commuters than among homemakers. These models were also used to conduct traffic strategy assessments. Various congestion and emission pricing schemes are, for instance, compared in Agarwal and Kickhöfer (2015).

Couplings between multi-agent traffic models and noise models are rarer. The first work was performed by Gerike et al. (2012), who coupled MATSim with the noise mapping software IMMI, although no numerical simulation example was given therein. Subsequently, Houot et al. (2015) coupled the MobiSim model with the MITHRA-GIS noise mapping 
software on a case study in Besançon (France) in order to test various scenarios: modification of the public transit offering, desynchronization of individuals' activity planning. Scenarios were analyzed in terms of the spatial distribution of sound levels for different time slots. Their study suggested that a small increase in the proportion of public transit exerts only a limited impact on the sound environment, while desynchronizing individuals' activity planning significantly modifies the temporal profile of the sound environment throughout the day. Kaddoura et al. (2017) proposed a coupling between MATSim and the RLS90 acoustic model, revealing the need to take the spatial and temporal variations of populations into account in exposure calculations. This modeling framework was then used in Kaddoura (2018) for a multi-criteria assessment involving traffic and noise indicators. This last study proved the benefit of policy-making that adopts a dynamic and differentiated approach whether or not the traffic is congested. The acoustic module of this modeling chain was updated in Kuehnel et al. (2019) and Zwick et al. (2021), updated noise prediction model in MATSim, that is RLS19. Kuehnel et al. (2020) proposed a method to estimate the effect of noise on housing prices based on this same coupling. Cucurachi et al. (2019) also relied on MATSim to evaluate the contribution of road transportation to the total noise footprint of land-based mobility. Lastly, from a decision-making perspective, Bean \& Joubert (2020) proposed a modeling approach based on MATSim to investigate offhour deliveries in Cape Town (South Africa); their work highlighted the need to account for the induced increase in noise pollution.

All these studies have shown the value of using multi-agent modeling to analyze acoustic impacts. In this paper, a complementary approach is introduced, more dedicated to an audience of acousticians. The aim is for this community to grasp the potential of multiagent traffic modeling in order to rise new research questions on acoustic modeling, on associations with annoyance, or on the estimation of specific acoustic indicators. Based on this premise, a module for reading and integrating MATSim outputs has been integrated into the NoiseModelling noise prediction tool, which is a free and open source tool, 
developed since 2010, and designed to produce environmental noise maps over very large urban areas that implements the CNOSSOS method (Commission Directive, 2015; Bocher et al., 2019; Aumond et al., 2020). Section 2 presents the resulting modeling chain. The potential of a modeling chain for use in exposure calculations and associated analysis is illustrated in Section 3 through examples from a case study in Nantes (France). Section 4 discusses the limitations and necessary further developments, while Section 5 offers a conclusion.

\section{Methods}

\subsection{Description of the modeling framework}

The method introduced herein couples a traffic simulation component with a noise modeling component. The first component is a multi-agent traffic simulation, MATSim (see Section 2.1.1 for a presentation), fed by inputs generated by the Eqasim component described in Section 2.1.2. The noise modeling component consists of the NoiseModelling software described in Section 2.1.3.

\subsubsection{Transportation modeling component}

The transport modeling component consists of the Multi-Agent Transport Simulation MATSim, as described in Horni et al. (2016). As is the case with other agent-based simulations, MATSim has been inspired by co-evolutionary principles. All agents seek to maximize their travel score while sharing the traffic network and its capacities with other agents. The map of all road segments is represented as a network graph, with roads being represented as links between two nodes and nodes serving as road intersections. The MATSim simulation cycle is depicted in Figure 1. The initial demand is derived from every agent's preferred schedule, i.e. where and when they wish to be within the simulated day. 
The mobility simulator (mobsim) is responsible for modeling the traffic interaction on the network. Several mobsim solvers are available, but the most common ones are based on a FIFO (First In, First Out) queue model. The principle herein is that every link has an input FIFO queue and an output FIFO queue. A vehicle can leave a link and enter the next one if it meets the following conditions:

It is the first vehicle in the output queue of the link scheduled to be exited;

It spends at least the minimum travel time allocated (max speed * length) on the link scheduled to be exited;

The link scheduled to be entered has not yet reached its maximum vehicle capacity.

The scoring part assigns a value to the agent's simulated trips. During the replanning phase, a given percentage of agents are allowed to alter their schedule, and mobsim is run again with these adjustments. The agents' ultimate purpose is to choose the trips with the best scores. After a desired number of iterations, MATSim outputs the agents' final schedule and traffic events for analysis.

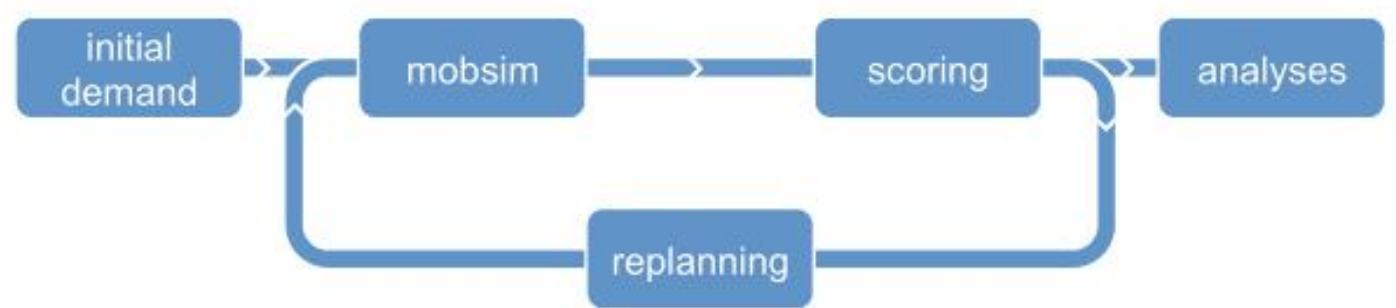

Fig. 1: MATSim simulation cycle (Horni et al., 2016)

To run a MATSim simulation, several input files are required, as described in Table 1 below. Table 1: MATSim inputs

\begin{tabular}{ll}
\hline File name & Contents \\
\hline network.xml.gz & The traffic network, a list of "links" with their \\
& geometry and a list of attributes (length, traffic \\
capacity, etc.).
\end{tabular}

persons.xml.gz

The list of agents with their daily schedules. 
activities.xml.gz

transit_schedule.xml.gz

config.xml
The list of all agents' "activities" and their locations, as defined in the agent's schedule.

The list of public transit (bus, train, tram, etc.).

The Simulation configuration file, setting the various parameters and locating the other files.

After running a MATSim simulation, a list of $\mathrm{xml}$ files containing the simulation output data is generated. The main output files used for coupling with NoiseModelling are listed in Table 2.

Table 2: MATSim outputs

\begin{tabular}{ll}
\hline File name & Content \\
\hline output_plans.xml.gz & The resulting daily schedules of all agents. \\
output_events.xml.gz & The list of traffic events.
\end{tabular}

MATSim events vary based on the mobsim solver, which is configured according to the type of transport used (e.g. public transit, car, bike). For example, events are generated by the simulation every time a vehicle moves from one road segment to another or every time an agent enters or exits a car or public transit vehicle. The list of events generated by a typical car trip is shown in Figure 2. 

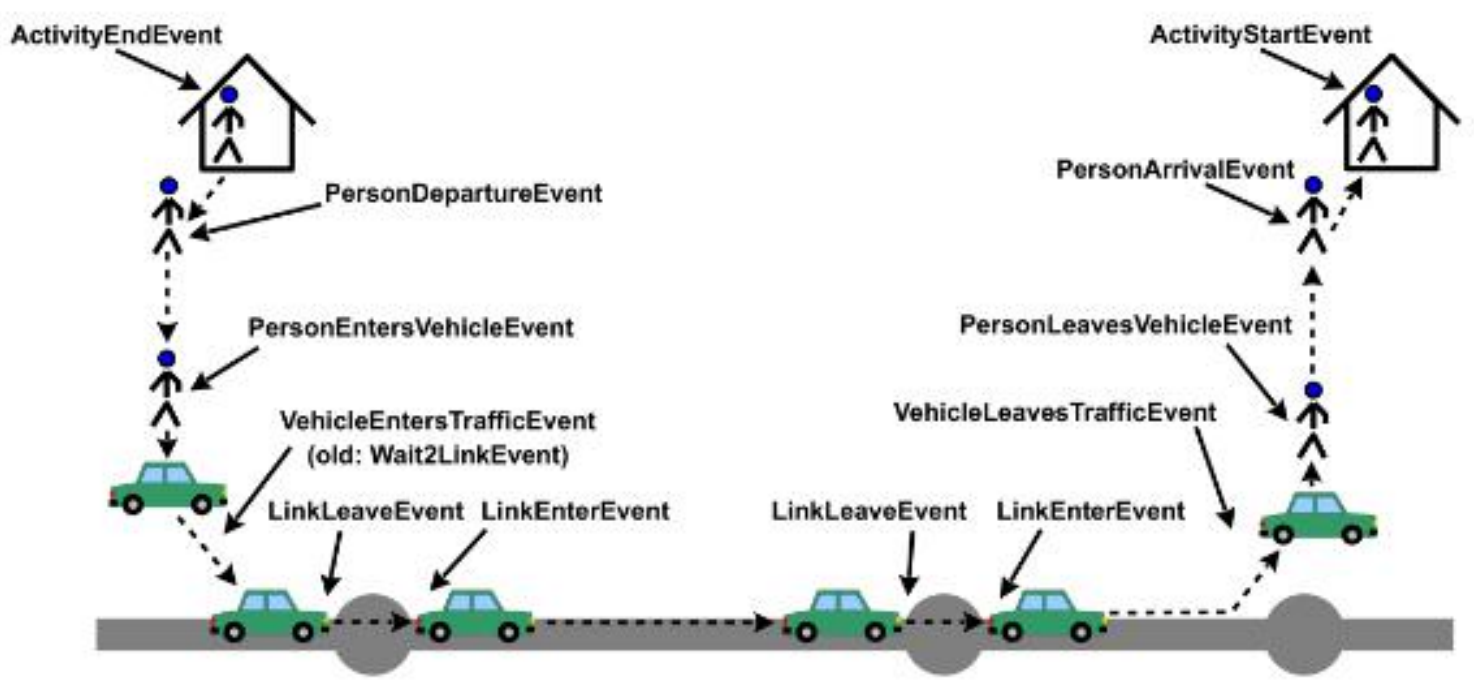

Fig. 2: MATSim events (Horni et al., 2016)

\subsubsection{The Eqasim project}

In order to prepare the MATSim simulation inputs, and more importantly a simplified microscopic representation of the actual population, the Eqasim project, as developed by Hörl and Balac (2020), can be used. This project consists of a modeling pipeline of data consumption and processing steps, making the transition from available public census data and other open source databases to a fully functional MATSim simulation in the French context. This project was chosen for the case study presented in Section 3 given its highprofile use in generating a MATSim scenario for the entire Paris Region (lle-de-France). Since France's census format is standardized, this project provided the most straightforward way to generate a valid traffic model.

Let's mention here that the MATSim scenarios generated by this pipeline involve a nonstandard approach to simulating traffic. More precisely, in Eqasim, the replanning phase of MATSim is replaced by a Discrete Mode Choice model that more heavily emphasizes mode change than on-time departure changes; this emphasis is typically reversed in the default MATSim model. 


\subsubsection{Noise modeling component}

The Noise modeling component of the modeling chain consists of NoiseModelling, which is a free and open source software developed primarily by the DECIDE team of the Lab-STICC (CNRS) and the Joint Research Unit in Environmental Acoustics UMRAE (Gustave Eiffel University) (Bocher et al., 2019; Aumond et al., 2020). The noise calculation method implemented within NoiseModelling is based on the standard European method CNOSSOS, which serves as a reference method to use for the Directive 2015/996 relative to environmental noise assessment and management (Commission Directive, 2015). The CNOSSOS model specifies, for different vehicle categories, the noise emission formulas to use to estimate the acoustic power relative to a given road traffic flow. The CNOSSOS model also specifies the noise propagation calculation method, describing the main physical phenomena affecting noise propagation: it includes methods for searching the acoustic rays between sources and receivers, reflections, diffractions, etc. The reader could refer to the Directive 2015/996 (Commission Directive, 2015) for a complete description of the model and its associated physical principles.

NoiseModelling is closely paired with spatial database H2GIS or PostGIS, given their large number of spatial functions and features. The calculation method, which relies heavily on GIS spatial data, is thus aligned with previous work that has demonstrated the contribution of GIS to calculating noise propagation via market software (Tang and Wang, 2007; Gulliver et al., 2015; Alesheikh and Omidvari, 2010; Reed et al., 2012).

This calculation is split into three modules:

- A noise emission calculation module estimates the sound power level based on input data, such as the mean flow rates and mean speeds per road segment, along with the traffic composition (ratio of medium, heavy and two-wheel vehicles);

- A path-finding algorithm searches the paths between sources and receivers, in considering the distance propagation, the number of reflections and diffractions (illustrated in Figure 3); 
- The software computes the attenuation value along each of the propagation paths for a selected range of octave bands, using the CNOSSOS-EU model.

The sound level at a receiver is the sum of the contributions of all sources; its output consists of a grid of receivers with an attributed sound level value computed in $\mathrm{dB}$.

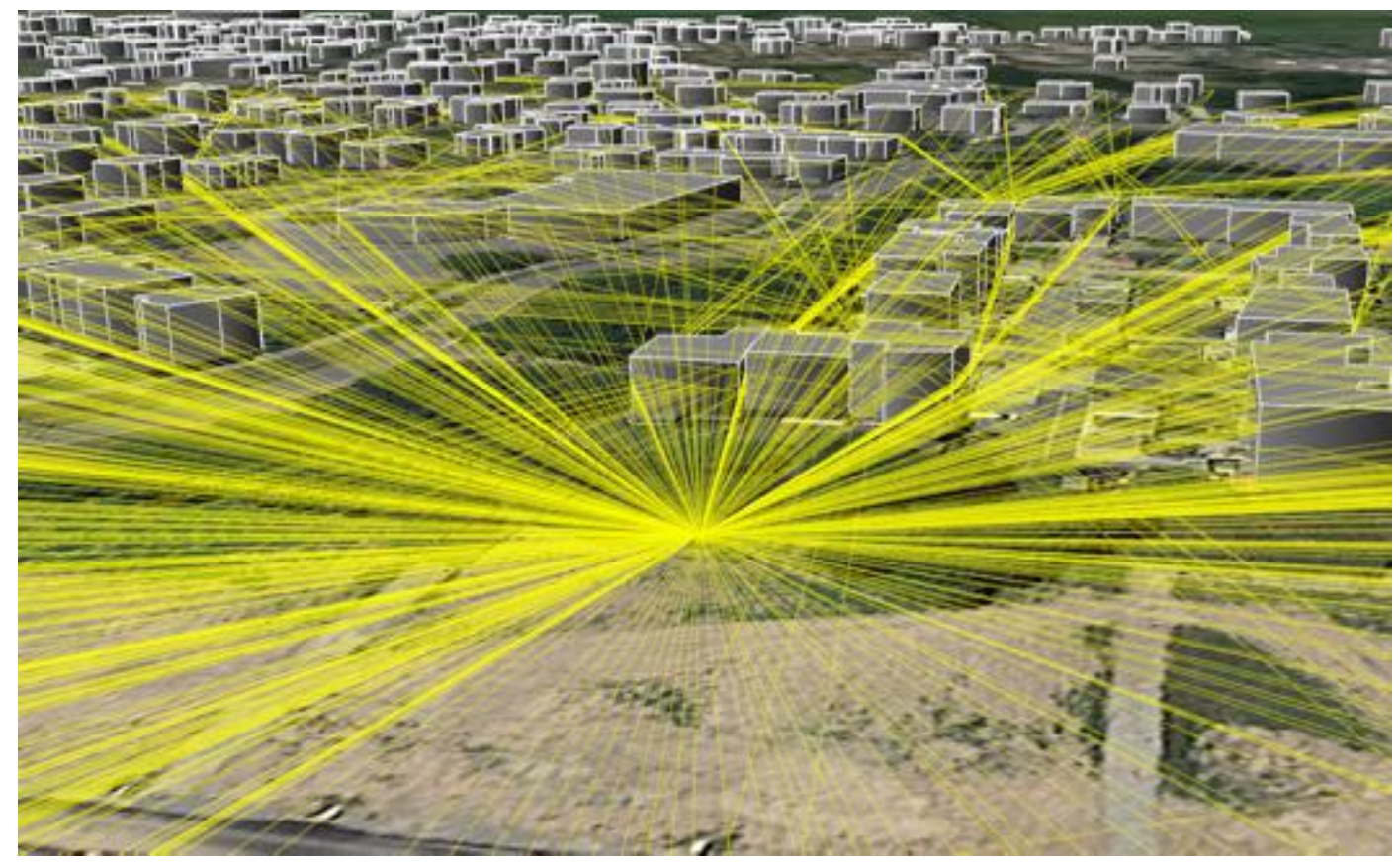

Fig. 3 : NoiseModelling ray tracing propagation implementation of the CNOSSOS method (source: noise-planet.org)

NoiseModelling was initially designed to produce environmental noise maps over large urban areas. The model has recently been coupled with the microscopic road traffic model SYMUVIA to estimate either the impact of local transportation strategies on noise dynamics (Can et al., 2019) or individual exposure at the local scale during commuting (Quintero et al., 2019).

\subsection{Coupling}

\subsubsection{General remarks}

The entire modeling framework is illustrated in Figure 4. The initial inputs are: 
Census data of the population to model;

Geographic data imported from the Open Street Map (OSM) project.

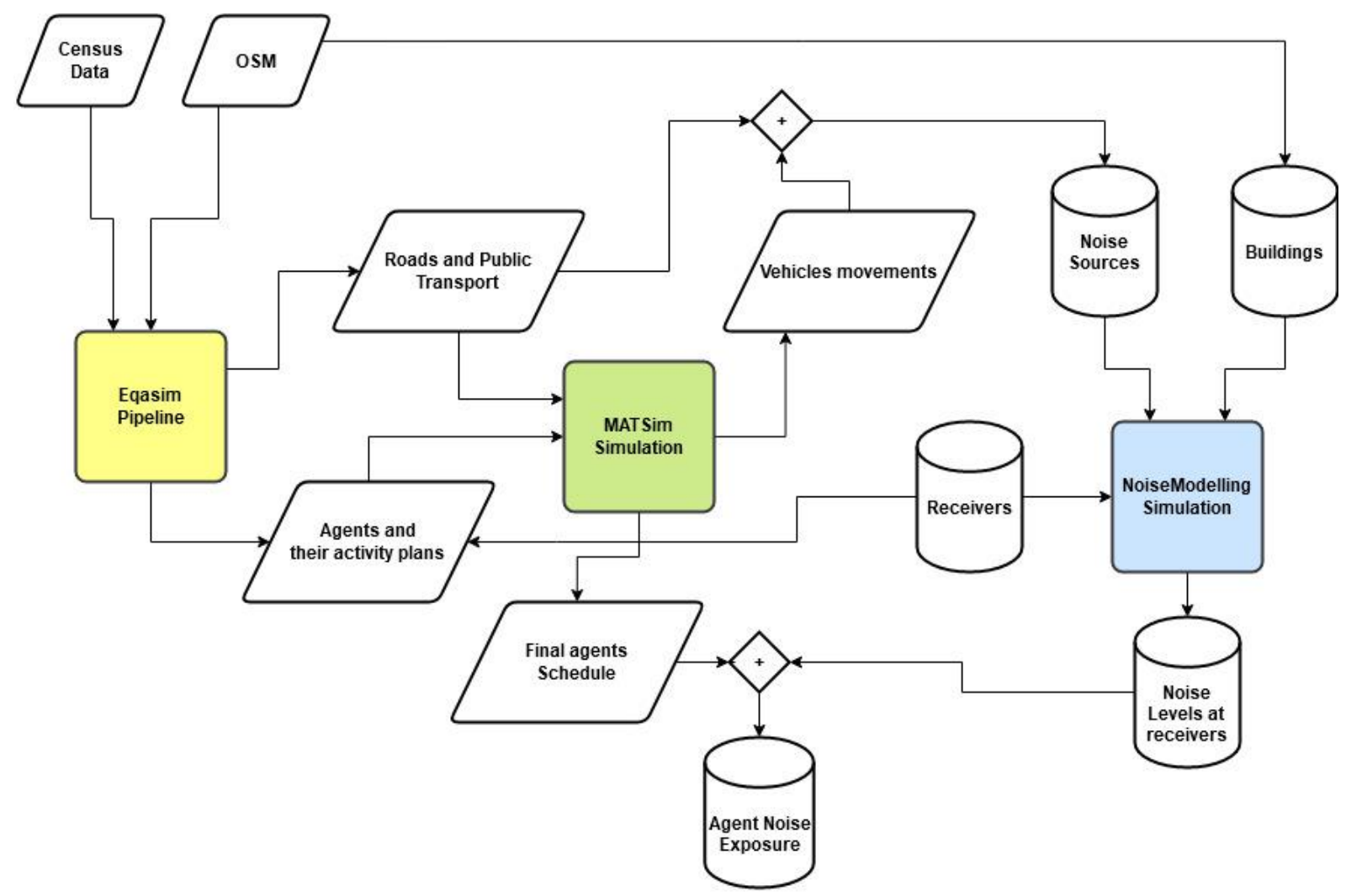

Fig. 4: Modeling framework

The Eqasim project uses these inputs in order to generate a synthetic population, directly usable in the MATSim multi-agent traffic simulation. More specifically, Eqasim is used to:

- generate a traffic network for the various transport modes such as car, train, bus, bike, etc;

- generate a list of agents, with characteristics such as age, gender, driver's license or household income;

- generate a list of a geographically placed activities (or facilities);

- generate a schedule, i.e. the sequence of activities that the agents will try to follow during the day, for every agent. Applying a procedure inspired by statistical 
matching algorithms, trip chains from household travel survey data are attached to the synthetic population (Hörl and Balac, 2020).

These inputs are then used to run a MATSim traffic simulation.

The MATSim simulation result is a list of events representing, among other things, vehicle movements. The elements in this list of benefit for noise calculations (time, position, vehicle, speed, etc.) are then extracted and used as noise sources in the noise modeling software.

Buildings are directly imported from the OSM database. Building height is taken from the OSM map and height is inferred based on available meta-data, such as the number of floors, when the height is not available. Noise receivers are determined from the agent's activity positions. The noise levels at the receiver positions can then be calculated. By importing the MATSim resulting agent schedules, the time and position of every agent is accessible. Agent noise exposures at any given time and throughout the simulated day can then be calculated.

The coupling steps and their key parameters are discussed in this section. The way time is handled, during the simulated day, is described in Section 2.2.2. The road traffic emissions calculation is described in Section 2.2.3. Sections 2.2.4, 2.2.5 and 2.2.6 cover respectively the receiver placement technique, the attenuation matrix calculation and custom implementations added to the framework.

\subsubsection{Time discretization}

Standardized noise maps in the European Union are built in considering 3 time periods, namely day, evening and night, respectively 6 am - 6 pm, 6 pm - 10 pm and 6 pm - 6 am for France. The input data for constructing these maps are given for each of these time periods; in most cases, they are typically given as annual averages. With MATSim, the state 
of the simulated traffic can be accessed at every time during the day, thus providing the opportunity to improve time resolution when generating traffic noise contribution maps.

Time periods can be managed in two ways. The first one is to average traffic data over the day, evening and night periods and then generate a noise map that is comparable to the European standard. The second way is to set a fixed time step, e.g. 1 hour, and generate a noise map for each one of these time steps, thus resulting in a finer time quantification for the traffic noise maps and leading to a new set of potential analyses requiring time variation.

In the work presented herein, the three following time discretization periods were implemented and can be chosen at runtime by the user: (i) day, evening, night; (ii) every hour; and (iii) every 15 minutes.

\subsubsection{Calculating road traffic noise emissions}

In CNOSSOS-EU, the source power level of a road segment is calculated based on the following parameters:

- Vehicle category;

- Number of vehicles per hour per category;

- $\quad$ Average speed per vehicle category.

Four vehicle categories serve to analyze road traffic, as defined in the CNOSSOS-EU model, i.e.:

- Category 1: Light-duty motor vehicles;

- Category 2: Medium heavy-duty vehicles;

- Category 3: Heavy-duty vehicles;

- Category 4: Powered two-wheelers. 
In the NoiseModelling software, noise sources are modeled as road segments with a vehicle flow per hour and an average speed per category. To extract vehicle flow and average speed per road segment (or network link), the enterLink and leaveLink events are extracted from the events output file (see Figure 2).

For each road segment, each vehicle type and each time period, the number of vehicles passing and their speed are stored. The average speed is then calculated and the NoiseModelling libraries are used to calculate a sound power level per meter for every road segment and every time period.

\subsubsection{Placing noise receivers}

In NoiseModelling, noise levels are calculated at the listed receiver's positions. This software offers various options for placing receivers. A new option has been added; it is specific to the coupling presented here and detailed in Section 2.2.6.2. Agents' activities, found in the activities.xml output file, can be imported into NoiseModelling in order to directly place receivers at the activities position, which in turn makes it possible to calculate noise levels in the different locations the agents may visit during the simulated day.

\subsubsection{Calculating the Source-Receiver noise attenuation matrix}

Once the receivers have been placed and the road sections discretized onto point sound sources, the source-receiver attenuation matrix is computed using the NoiseModelling libraries. For each source-receiver couple, this matrix stores the noise attenuation, which encompasses all the various noise propagation paths between source and receiver.

More specifically, for each receiver, a path-finding algorithm locates the list of contributing noise sources, along with their respective attenuation. In practice, this attenuation represents the arithmetic difference between the sound power level of the 
source ( $\mathrm{Lw}$, in decibels) and the pressure level at the receiver (Lp, in decibels). This matrix is calculated once and stored in the NoiseModelling database.

As a result, several noise maps can be calculated just by modifying the power level of the sources, for instance in the event average traffic speeds are modified. For this step, the new contribution of a sound source at a given receiver is obtained by simply subtracting the source receiver attenuation from the new source power level.

The last stage entails using the attenuation matrix with the road segment noise power level to calculate, for each receiver and for each time period, a resulting sound pressure level. A noise map is thus obtained from a MATSim simulation output.

\subsubsection{Integration of the coupling}

Such a coupling was performed between MATSim version 11.0 and NoiseModelling version 3.3. It was decided to integrate the coupling inside NoiseModelling, in the form of a list of MATSim-related scripts. Since both MATSim and NoiseModelling are written in Java programming language, this coupling was accomplished using this same language. This common language was particularly useful for creating import scripts, by using the MATSim library as a dependency in NoiseModelling. The coupling code was released in version 3.3.2 of NoiseModelling, along with a specific tutorial (https: //noisemodelling.readthedocs.io/en/latest/Matsim_Tutorial.html) and can be found on the project Gihub repository: https://github.com/Ifsttar/NoiseModelling.

An example of a script is shown in Figure 5, using the NoiseModelling GUI tool, WPSBuilder. 

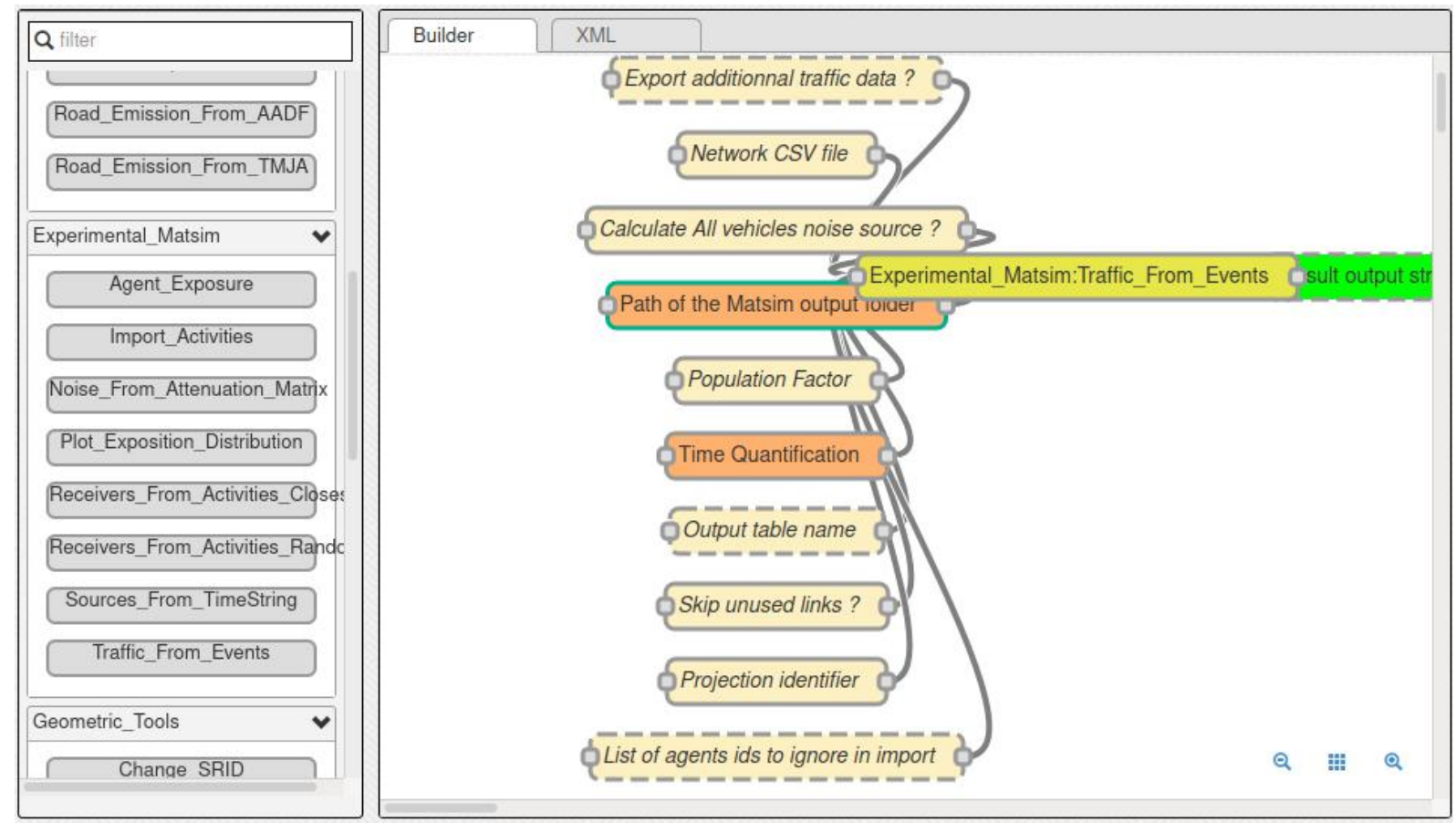

Fig. 5: A NoiseModelling script that imports traffic data from MATSim

Some of the specific developments achieved during the coupling are detailed in Sections 2.2.6.1 and 2.2.6.2, focusing respectively on the importation of roads and receiver placement.

\subsubsection{Maintaining road segment geometries}

One of the issues involved when coupling the two models pertains to road geometries. In MATSim, when importing an Open Street Map (OSM) file, the roads are simplified, as illustrated in Figure 6. 


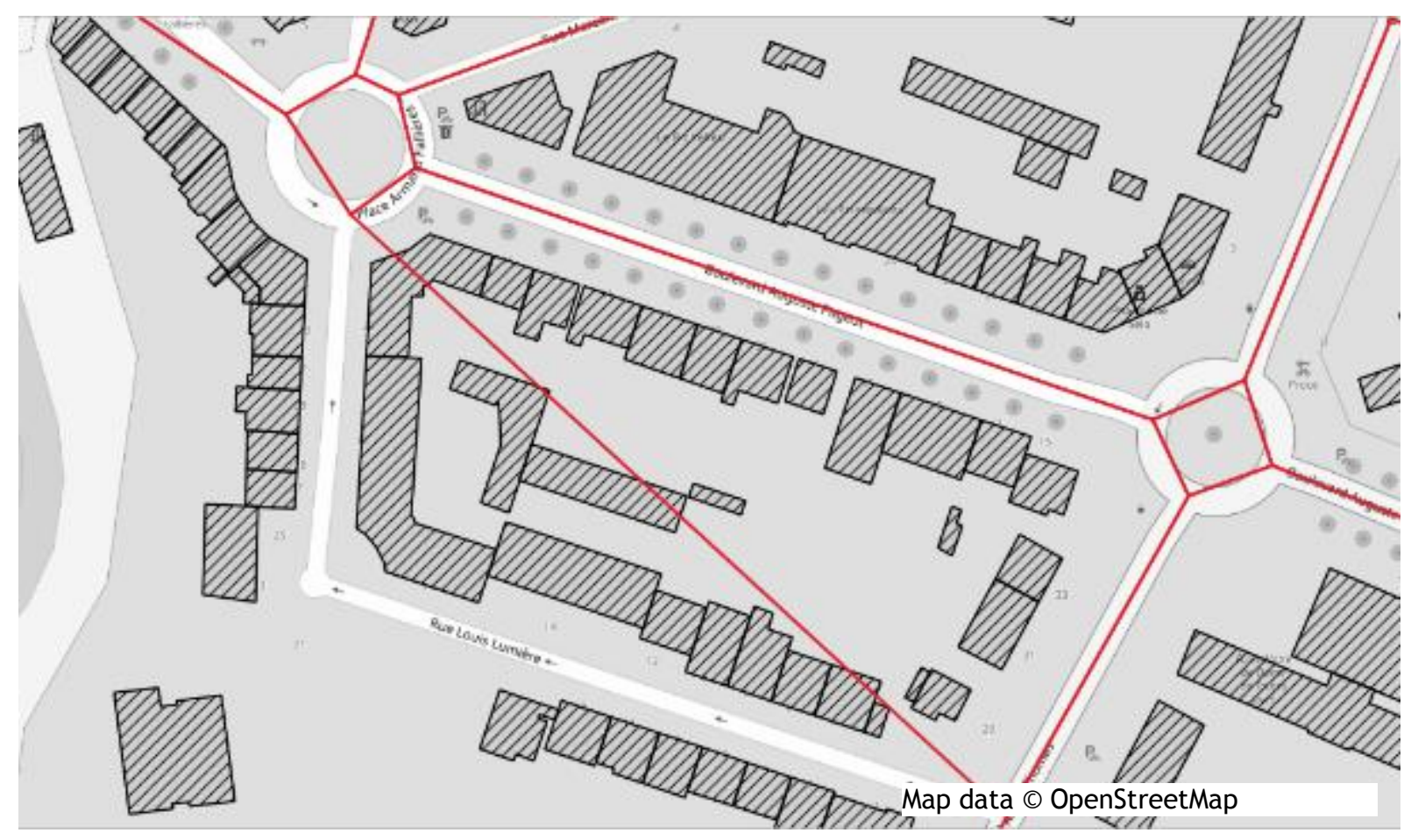

Fig. 6: Road simplification in MATSim

This simplification is carried out in MATSim to limit network size, and thus the computational cost of the simulation. Most OSM map import codes replace curved roads with straight lines between each network intersection, while saving the total length of the curved street as additional data. This simplified network of links is illustrated using red lines in Figure 6. An issue arises if these links are directly used as a noise source in NoiseModelling for two reasons affecting the sound propagation calculation step: 1) the simplified network can pass through buildings; and 2) the distance from the source to a fixed receiver can vary significantly. Both cases are illustrated in Figure 6.

To overcome this particular issue, a list of original geometries is stored in a CSV file when importing the network in MATSim, prior to link simplifications. This geometry file is then used, when importing the MATsim traffic output into NoiseModelling, to replace the simplified roads before the NoiseModelling propagation calculation .

2.2.6.2 Snapping Activity Receivers to Buildings 
Snapping activity receivers to buildings is a crucial step since source-receiver distances have an important impact on the resulting noise levels. Here one wants to comply as much as possible with the existing exposure calculation standard methods, so that comparing in a further work the obtained results under different modeling approaches or different mobility policies would be possible.

When generated by the Eqasim pipeline, version 1.1.0, agent activities are placed using postal addresses. The pipeline actually uses a publicly available database provided by The National Institute of Geographic and Forest Information (IGN), i.e. BD-TOPO, to assign activities to a proper address. For example, an "education" activity location is chosen amongst schools or universities addresses. The problem here is that the actual position of the activity is placed near the front door of every building. So, if these locations are used directly as receiver positions, the resulting source-receiver distances would not correspond to the equivalent for standardized noise maps. A second issue is that the receivers would almost always be the most exposed to the traffic noise.

As a consequence, to calculate noise level at the activities in a way comparable to that of the standardized noise maps, the agents' activity locations are "snapped" to buildings, according to the following method:

All noise receiver points that would be placed around buildings, should it be possible to evaluate the standard noise exposure, are generated with a NoiseModelling script. In following Directive 2002/49/EC of the European Parliament and European Council of the 25 June 2002 Standard (EC 2002), receivers are placed around buildings 4 meters above ground, 2 meters from the facade and 5 meters from one another (Figure 7).

Next, for each activity, the closest building is identified and a random receiver is chosen amongst the receivers that surround the chosen building. The receiver's position is assigned to the activity. A maximum distance of 200 meters is set, 
beyond which the activity's position remains the same apart from height, which is imposed at 4 meters.

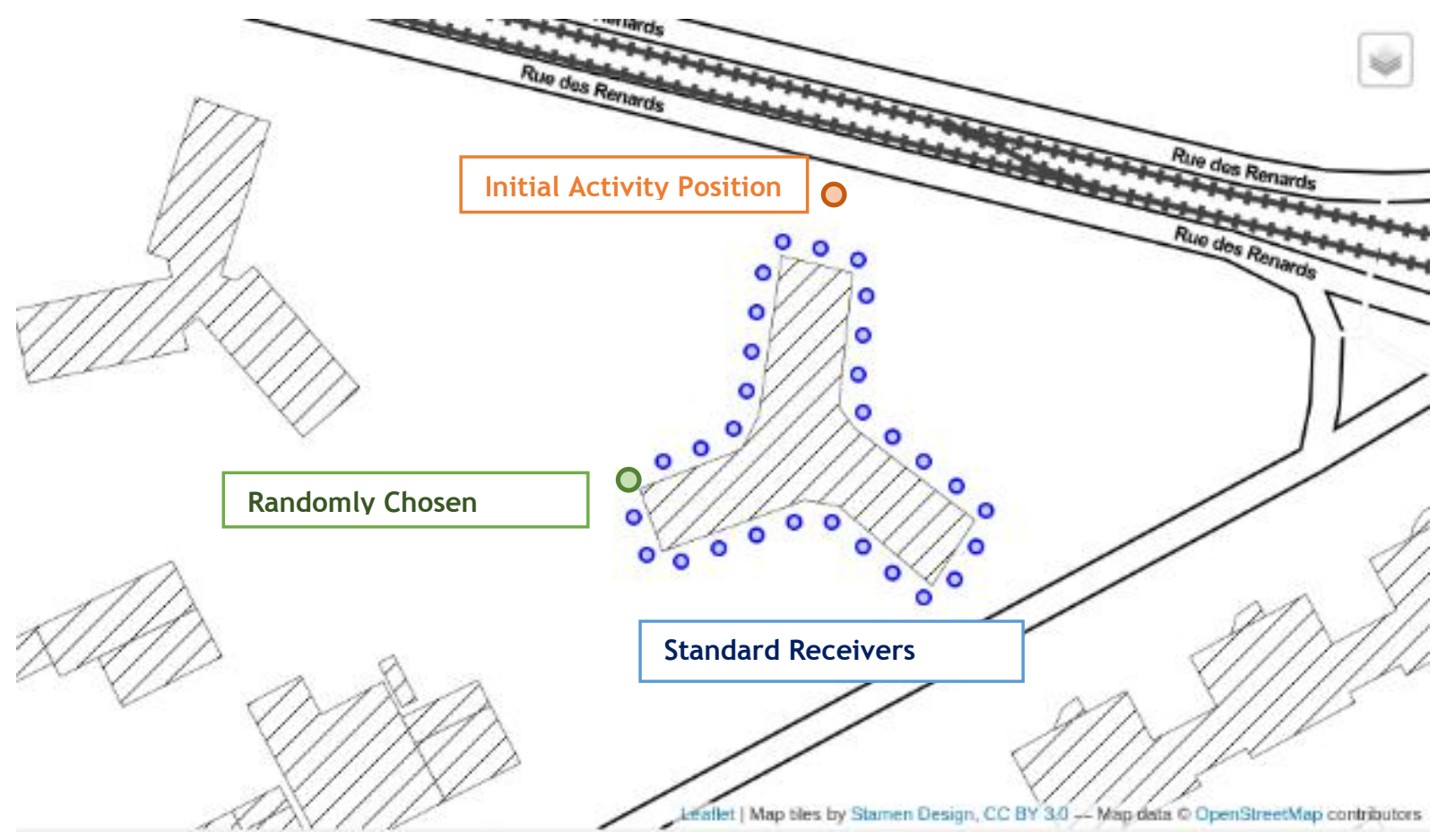

Fig. 7: Placement of the NoiseModelling receiver based on the agent's activity positions Note that with this method for assigning agents to buildings, the capacity of buildings is not a used variable, at least not directly. The exact method was not analyzed here and is outside the scope of our work, so it is not impossible to have activities that locally exceed the capacity of buildings, even if this might be rare.

\section{Case study}

A MATSim simulation was set up for Nantes (France) and 10 surrounding cities as a proof of concept to illustrate how the proposed modeling framework provides a workable system for refining the assessment of individual exposure to road traffic noise. The NoiseModelling software was used to calculate the agent-based noise levels, according to the method described in Section CNOSSOS-EU emission and propagation standard.

\subsection{Description of the study area}


The study area is shown in Figure 8 . Nantes is the France's sixth-largest city, with a population of 303,382 . The entire study area contains 522,008 inhabitants (according to the latest census data) for a surface area of $317.5 \mathrm{~km}^{2}$ (i.e. population density $=1.645$ people $/ \mathrm{km}^{2}$ ); it has been divided into 206 distinct IRIS (i.e. average population per IRIS = 2,534). ${ }^{1}$ Municipalities with over 10,000 population plus a large proportion of those with 5,000 to 10,000 population have been divided into several IRIS units and, by extension, all municipalities not divided into IRIS units do constitute IRIS units in themselves.

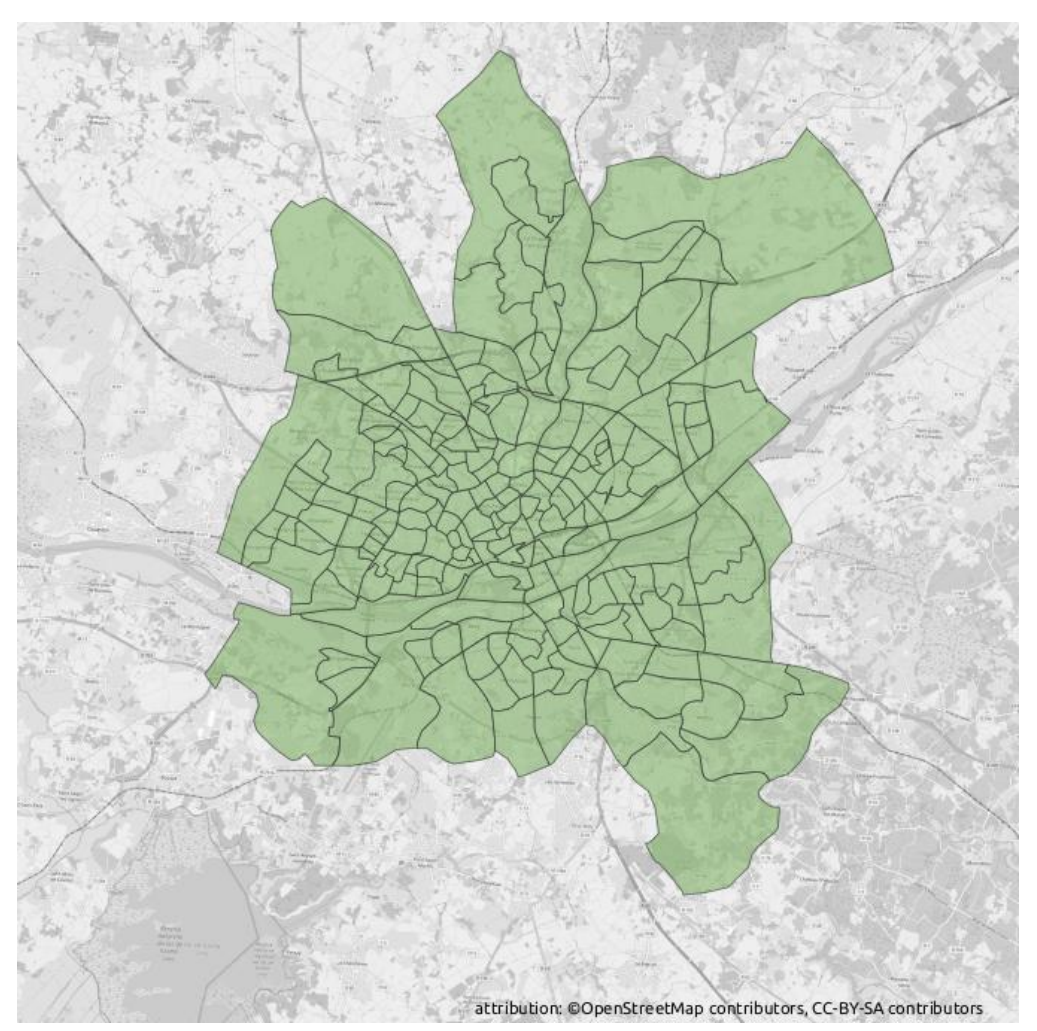

Fig. 8: The municipalities chosen for the Nantes case study, and their IRIS blocs

For practical purposes, e.g. reducing computation time, smaller population samples (i.e. lower fraction of travelers) are used as input in most agent-based simulations. The Eqasim population scaling factor parameter, and hence the MATSim population scaling factor, is set at 25\%; network link parameters are adjusted by MATSim accordingly. Within the study

\footnotetext{
1 In French, IRIS is an acronym of "aggregated units for statistical information".
} 
area, this scaling corresponded to 130,496 agents distributed across 67,021 households. The agents were ascribed activity plans generated by Eqasim, which corresponded to a total of 476,823 trips during the simulated day.

The resulting traffic was analyzed in 15-minute time periods. The NoiseModelling propagation was computed with the following parameters:

Maximum source distance: 750 meters;

Maximum reflection distance: 50 meters;

Reflection order: 1;

Vertical diffraction: enabled;

Horizontal diffraction: enabled.

To have an approximate idea of the total computation load, the different steps and their corresponding computation time are presented below for a 14 -core, $3.6 \mathrm{GHz}, 48 \mathrm{Go}$ of Ram Ubuntu virtual machine:

Eqasim synthetic population: 2 hours;

MATSim simulation: 4 hours;

NoiseModelling simulation: 5 hours.

\subsection{Results}




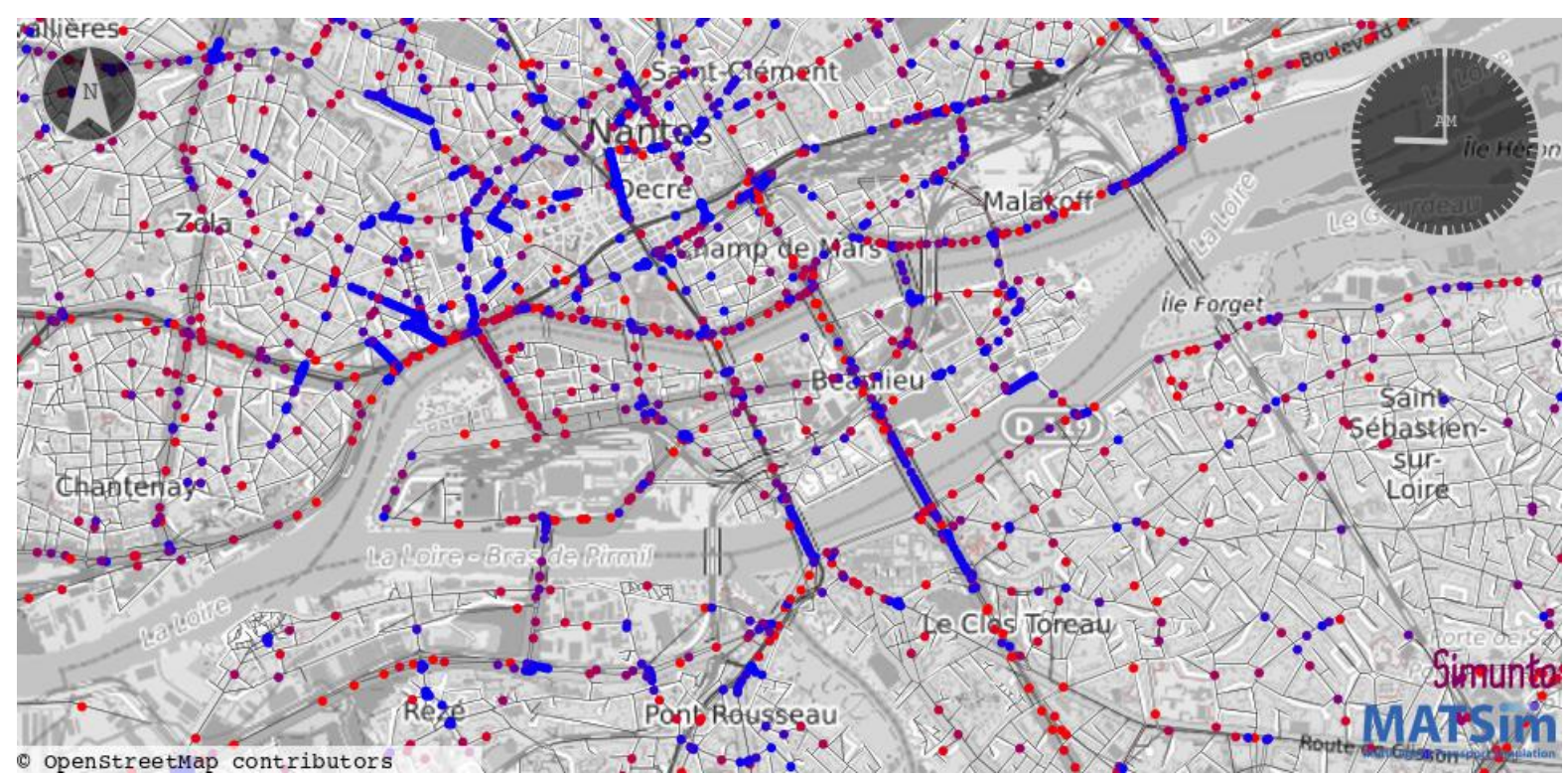

Fig. 9: Nantes area vehicles colored according to their speed (red: fast, blue: slow)

Figure 9 illustrates the simulated vehicles resulting from the MATSim simulation, around the city center, at 9:00 am of the simulated day. The figure highlights areas of traffic slowdown within the area, particularly around certain intersections, due to high travel demand during this time slot. From this point forward, the previously presented framework is used to obtain noise levels per time period at each agent activities position. Each of the 96 computed noise maps corresponds to a 15-minute period during the simulated day. Such maps are shown in Figures 10 and 11. This figure classically indicates the high noise levels along the main roads, especially the ring road. The originality of this approach lies in considering the agents' position over the pertinent time slot, for purposes of both calculating noise levels and determining agents' exposure. This approach makes it possible to: (i) calculate the temporal evolution of noise levels throughout the day; and (ii) refine the calculation of agent exposure, by means of overlapping the dynamic noise map and the agents' position. 




Fig. 10: Noise levels, at agent activities positions, between 9 am and 9:15 am 


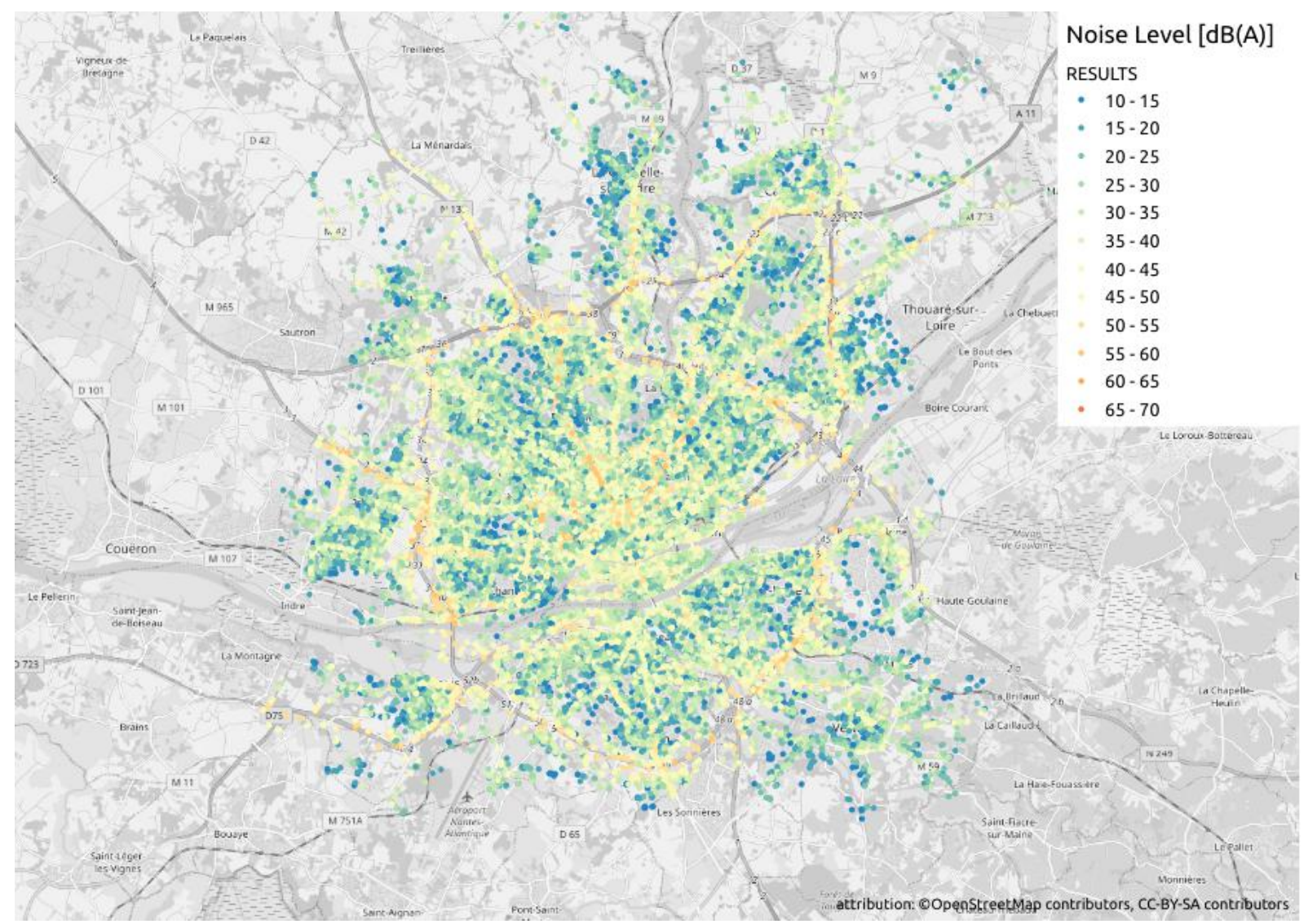

Fig. 11: Noise levels, at agent activities positions, between $11 \mathrm{pm}$ and 11:15 pm

From an agent's perspective, in moving from activity to activity throughout the daily plan, a noise level can be assigned every 15 minutes. The noise levels received by an agent throughout the day are computed by summing all 15-minute noise levels depending on the daily activities. Similarly, the noise level at an agent's "home" activity can be computed. This "home" activity noise level is comparable to those that would have been obtained from a standardized static noise map. Indeed, the classical method for calculating noise exposure assigns city dwellers to their place of residence. Both noise level exposures are plotted in Figures 12 and 13 for a "worker" and "student", respectively.

The dose level plotted in these figures represents the cumulative noise exposure level of the agent-based or home-based activity. This value can only increase and its final value, at the end of the day, equals the equivalent noise level over the course of this same day. 
Agentld : 228798

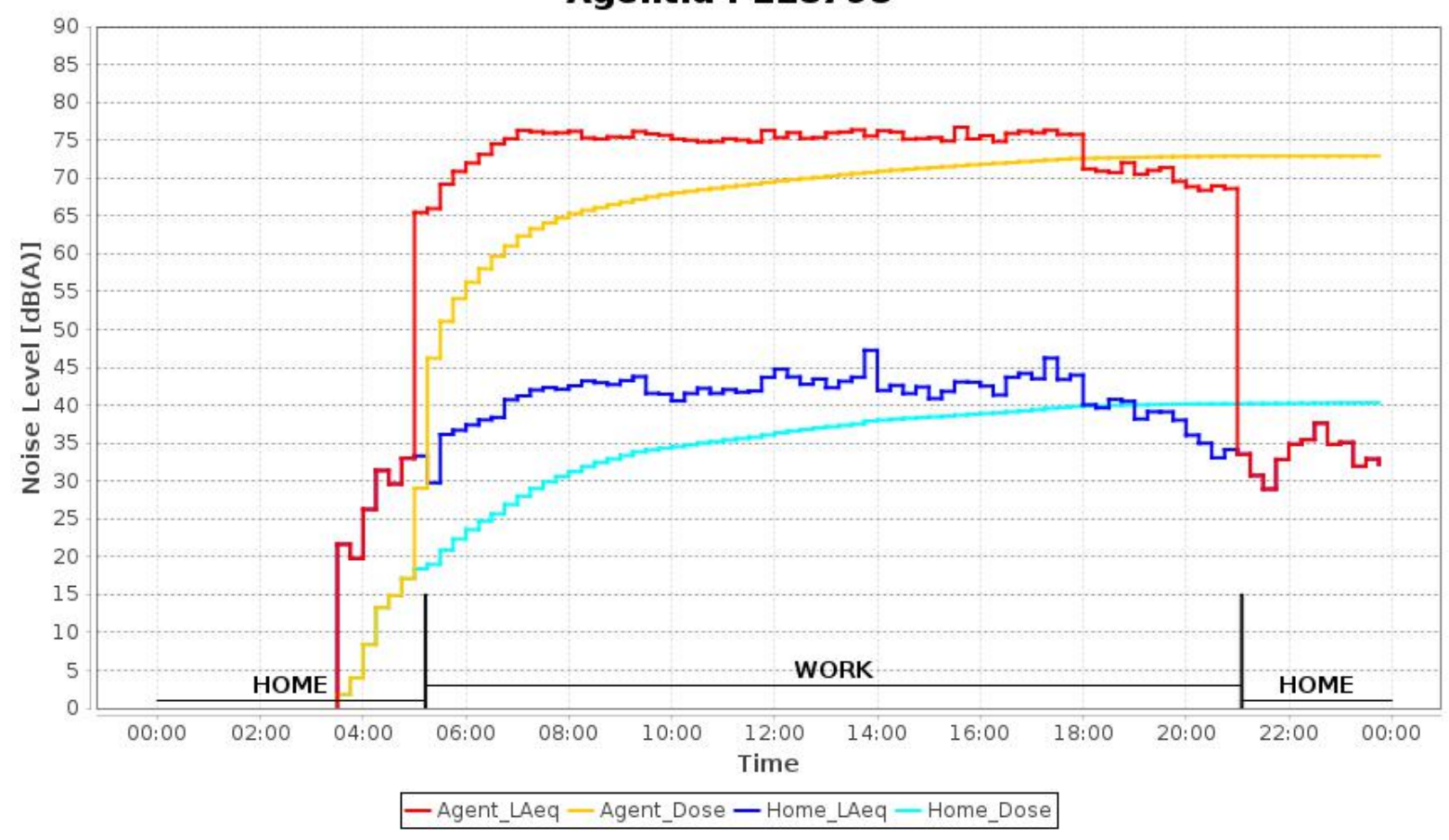

Fig. 12: Evolution in exposure of a "worker" agent during the day

The agent depicted in Figure 12 has a worker-type activity plan: at home until 5:30 am, at work until 9 pm, and then back home again after 9 pm. The two curves Agent_LAeq and Home_LAeq are superimposed until 5:30 am since the agent is at home. Afterwards, the two curves diverge, with the agent being exposed to higher noise levels at the workplace than what would have been received had the agent stayed home. This curve shape is typical of an agent who lives outside the city center where noise levels are low, while working at a location with high noise pollution. The agent-based noise exposure calculation indicates that this agent is exposed to approx. $73 \mathrm{~dB}(\mathrm{~A})$ daily, whereas a standard noise map would only consider the home exposure of $40 \mathrm{~dB}(\mathrm{~A})$. This discrepancy is accentuated by the energy averages used in acoustics: the daily noise dose is heavily influenced by the time slots during the day when the agent is exposed to high noise levels. 


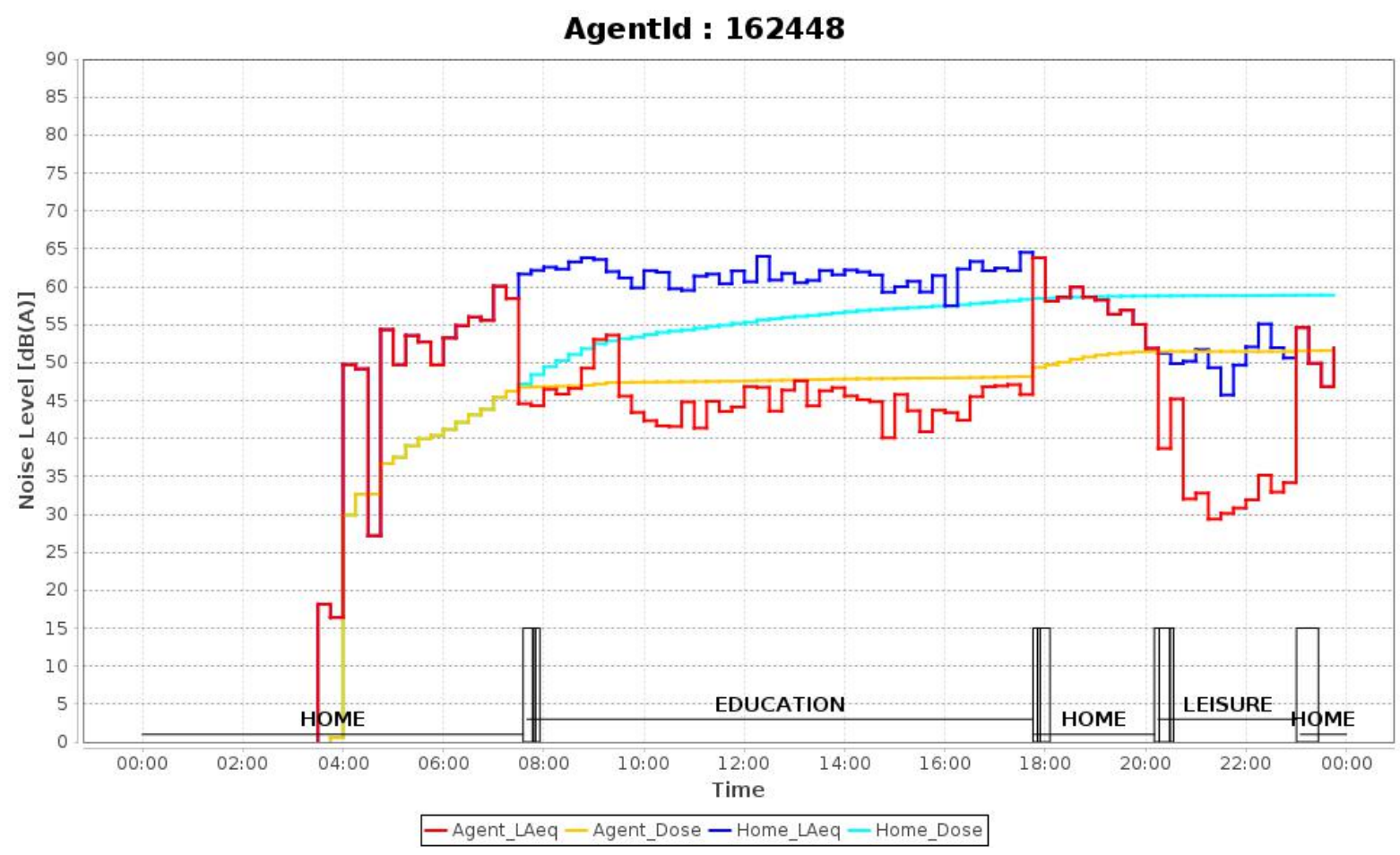

Fig. 13: Evolution in exposure of a "student" agent during the day

The agent depicted in Figure 13 follows a typical student activity plan: at home until 8 am, at school or university between 8 am and $6 \mathrm{pm}$, back home between $6 \mathrm{pm}$ and $8 \mathrm{pm}$, out of the house from $8 \mathrm{pm}$ until $11 \mathrm{pm}$, and then home after that. Here, the two curves Agent_LAeq and Home_LAeq only differ between 6 am and 6 pm and between 8 pm and 11 pm. The agent is exposed to lower road traffic noise levels at the place of education than what would have been received by staying at home. This curve shape is typical of an agent living in a noisy environment (e.g. near the ring road), while working in a less noisy site, e.g. a university campus. In this example, the agent's leisure activity takes place in a very quiet location. Consequently, the agent-based noise exposure calculation indicates that this agent is exposed to around $52 \mathrm{~dB}(\mathrm{~A})$, whereas a standard noise map would only consider the home exposure of $59 \mathrm{~dB}(\mathrm{~A})$.

These two examples illustrate both the limitations of conventional methods for calculating noise exposure based solely on place of residence and the possibility of examining in greater detail the impact of mobility on noise exposure. 


\section{Discussion}

We are proposing a multi-agent noise modeling framework that relies on open source tools and data. The modeling framework presented comprises two distinct modeling systems: a multi-agent traffic simulation component, MATSim, and a noise modeling component, NoiseModelling. The application of this framework to a case study has illustrated its relevance when considering an assessment of the dynamic exposure of individuals to road traffic noise. However, outputs from both models are characterized by a variety of uncertainties, both of which are capable of affecting the exposure assessment. In addition, this approach encounters limitations that will need to be addressed in the future.

The following limitations related to the traffic simulation component for estimating noise exposure have been identified:

As regards the multi-agent traffic simulation component, Zughe et al. (2019) showed the population scaling factor to be the most influential parameter in MATSim simulations. Some authors (Hörl and Balac, 2020; Ben-Dor et al., 2020, 2021; Zughe et al., 2019) investigated the sampling rate at which reliable simulation results can be expected; however, studies of downscaling implications when assessing individuals' exposure to traffic noise are still lacking. Even if a downscaled population could be considered reasonable from the standpoint of traffic simulation, uncertainty remains regarding the reliability of the noise exposure calculation stemming from the simulation. Most studies previously conducted (e.g. Kaddoura et al., 2017) used a scaling factor of 0.1 and did not include a sensitivity analysis for this parameter. Without further information, a scaling factor of 0.25 has been chosen for this study; however, undertaking a study with a scaling factor of 1 (i.e. considering all agents) is feasible for a city the size of Nantes. 
The modeling framework adopts a representation of sound sources in the form of a traffic flow described per road segment by means of a flow rate and average speed. This approach can lead to discrepancies near intersections, where vehicle speeds are not uniform and where acceleration phases affect emissions (Can and Aumond, 2018). Errors can reach $4 \mathrm{~dB}(\mathrm{~A})$ for highly congested road segments yet remain limited under free-flow conditions (Can and Botteldooren, 2011). It is quite difficult however to assess the benefit of representing sound sources in the form of vehicle trajectories, thus providing access to the distributions of speeds and accelerations practiced by the vehicles on the network. The flow model integrated into MATSim is not in fact intended to finely replicate the vehicle trajectories.

Since MATSim is not a microscopic modeling framework, it simplifies the vehicle kinematics. Lane changes, acceleration phases, driver decisions, overtaking or adaptative signal controls are just some of the many traffic phenomena not modeled herein. This shortcoming could be mitigated to some degree by stochastically adding dynamics to the traffic flow between the MATSim and NoiseModelling simulations. Acceleration profiles, speed randomization or overtaking can, to some extent, be added to the coupling method in order to better describe the kinematic parameters with an impact on noise emissions. Let's note that integrating these dynamic phenomena would not be compatible with the CNOSSOS-EU model but instead more similar to the approach followed within previous couplings that have relied on microscopic traffic software. These improvements could build upon the probabilistic approach proposed by Aumond et al. (2018) to assess sound level distribution, in which vehicle positions are randomly chosen based on road traffic characteristics. Another possibility would be to locally complement the modeling with the outputs of a microscopic road traffic model, as it is done in Chevallier et al. (2019) and Estevez-Mauriz et al. (2019). This would 
enable estimating dynamic noise indicators but entails calibration and downscaling issues.

Lastly, a few limitations are specific to the Eqasim Project when using it to compute noise emissions, namely:

- Freight traffic is not generated. Moreover, freight vehicles mainly belong to the $2^{\text {nd }}$ and $3^{\text {rd }}$ CNOSSOS vehicle categories, which implies that only public transit buses are present in these categories.

- No motorbikes, i.e. the $4^{\text {th }}$ CNOSSOS vehicles category, are present; hence, they cannot be used in NoiseModelling.

These limitations are anticipated to be overcome in future work.

The following limitations related to the noise simulation component for estimating noise exposure have been identified:

As regards the noise component, the way receivers are placed in the NoiseModelling simulation exerts a tremendous impact on results. If an activity receiver is placed on the street side of a building, it will have a much higher noise exposure value than if the same activity receiver is placed on its shady side. Relying on how the Eqasim Project generates activity positions, i.e. randomly inside city districts, and then snapping this position onto buildings tends to randomize these differences. Yet a more elaborate way of placing activity receivers may be worth implementing. At the cost of increased computation times, a set of receivers could be created around the building and averaged in order to provide a more building-centered approach to activities-based noise exposure.

The modeling framework considers exposures with an exclusive focus on road transport noise, whereby impacts are expressed through energy-averaged indicators. Although recommended by calculation standards, this approach does not fully reflect the acoustic impacts potentially observed in urban areas. Gille et al. (2016) 
highlighted that two-wheeled vehicles and the strong temporal variations in noise levels they induced were perceived very negatively. Moreover, the effects are nonlinear even with aggregated indicators. The relationships built to link noise annoyance to long-term noise levels have a polynomial form (Miedema and Vos, 1998). In addition, research on soundscape has reached a consensus regarding the importance of preserving quiet urban neighborhoods, with methods for their identification and preservation described in EEA (2014). The context is also of great importance. Rey Gozalo and Barrigon Morillas (2017) showed that sound environments in quiet neighborhoods are generally perceived differently (more positive) for passers-by than for residents.

Lastly, as regards the exposure calculation, the current implementation ignores agents' exposure during trips since it focuses on activities. To fully address agents' actual exposure, it will be necessary to rely on experimental campaign data to understand the factors between levels calculated on the street and actual exposure by type of transport or activity.

\section{Conclusion}

When assessing the impact of traffic-generated noise on populations, an examination of population activity throughout the day, instead of considering just their home building exposure, can lead to very different conclusions. Agent-based traffic and noise modeling software can be used to evaluate such differences.

The multi-agent noise modeling framework presented in this paper has been assembled by coupling open source tools, namely the Eqasim Project, the MATSim multi-agent transport simulator and the NoiseModelling software implementing the CNOSSOS-EU emission and propagation standard, thus taking full advantage of the development work carried out within the two communities and ensuring a reproducibility and extensibility. The coupling was integrated within NoiseModelling, in the form of a list of MATSim-related scripts. 
The proposed framework opens new perspectives in road traffic noise analysis. For instance, it is now possible to conduct an evaluation of the traffic noise impact on certain categories of agents, based for example on their social attributes or locations of residence and activity, with the symmetrical advantage of being able to evaluate the impact of a certain category of agents on global traffic noise emissions. The benefits of the tools and methods from NoiseModelling also enable the acoustics community to enhance the sound environment characterization through for instance specific noise indicators calculation.

\section{Acknowledgements}

This work is part of the MUTANDIS project (Innovative solutions for sustainable mobility in periurban areas), funded by the I-sSite Future.

\section{References}

Agarwal, A., Kickhöfer, B., 2015. Agent-based Simultaneous Optimization of Congestion and Air Pollution: A Real-World Case Study. Procedia Computer Science 52, 914-919.

Alesheikh, A.A., Omidvari, M., 2010. Application of GIS in Urban Traffic Noise Pollution. International Journal of Occupational Hygiene 2(2), 79-84.

Andersson, H., Jonsson, L., Ögren, M., 2010. Property Prices and Exposure to Multiple Noise Sources: Hedonic Regression with Road and Railway Noise. Environmental and Resource Economics 45(1), 73-89.

Aumond, P., Jacquesson, L., Can, A., 2018. Probabilistic modeling framework for multisource sound mapping. Appl. Acoust. 139, 34-43. doi: 10.1016/j.apacoust.2018.04.017.

Aumond, P., Fortin, N., Can, A., 2020. Overview of the NoiseModelling open-source software version 3 and its applications, Proceedings of the 49th International Congress and Exhibition on Noise Control Engineering e-congress, 20-23 Aout 2020, Seoul, South Korea. 
Barreiro, J., Sánchez, M., Viladrich-Grau, M., 2005. How much are people willing to pay for silence? Applied Economics 37(11), 1233-1246.

Bean W.L., Joubert J.W., 2020. A case study of off-hour delivery collaboration and costsharing between freight receivers and carriers. Transportation Research Procedia 46, 141148.

Ben-Dor, G., Ben-Elia, E. Benenson, I., 2020. Spatial implications of Population Downscaling: A MATSim Study of Sioux Falls Morning Peak Traffic. Procedia Computer Science 170, 720-725.

Ben-Dor, G., Ben-Elia, E. Benenson, I., 2021. Population downscaling in multi-agent transportation simulations: A review and case study. Simulation Modelling Practice and Theory 108, doi: 10.1016/j.simpat.2020.102233

Bocher, E., Guillaume, G., Picaut, J., Petit, G., Fortin, N., 2019. NoiseModelling: An Open Source GIS Based Tool to Produce Environmental Noise Maps. ISPRS International Journal of Geo-Information, MDPI, 8(3), pp.130. 〈10.3390/ijgi8030130〉. 〈hal-02057736〉

Braubach, M., Fairburn, J., 2010. Social inequities in environmental risks associated with housing and residential location-a review of evidence. Eur J Public Health 20, 36-42.

Can, A., Chevallier, E., Nadji, M., Leclercq, L., 2010. Dynamic traffic modeling for noise impact assessment of traffic strategies. Acta Acustica united with Acustica 96(3), 482-493.

Can A., Botteldooren, D., 2011. Towards traffic situation noise emission models. Acta Acustica united with Acustica 97(5), 900-903.

Can, A., Aumond, P., 2018. Estimation of road traffic noise emissions: the influence of speed and acceleration. Transportation Research Part D 58, 155-171.

Can, A., Aumond, P., Bécarie, C., Leclercq, L., 2019. Dynamic approach for the study of the spatial impact of road traffic noise at peak hours, Proceedings of the 23rd International Congress on Acoustics, Aachen, Allemagne, 09-13 September, 2019. 
Chaix, B., 2018. Mobile Sensing in Environmental Health and Neighborhood Research. Annu Rev Public Health 39, 367-384.

Chevallier, E., Can, A., Nadji, M., Leclercq, L. 2009. Improving noise assessment at intersections by modeling traffic dynamics. Transport. Res. Part D: Transp. Environ. 14(2), 100-110.

Commission Directive (EU) 2015/996 of 19 May 2015 establishing common noise assessment methods according to Directive 2002/49/EC of the European Parliament and of the Council (Text with EEA relevance), vol. OJ L. 2015.

Cucurachi, S., Schiess, S., Froemelt, A. Hellweg, S., 2019. Noise footprint from personal land-based mobility. Journal of Industrial Ecology 23(5), 1028-1038.

De Coensel, B., Can, A., Madireddy, M., De Vlieger, I., Botteldooren, D., 2010. Combined assessment of noise and air pollution caused by road traffic. Proceedings of the Institute of Acoustics \& Belgium Acoustical Society, Noise in the Built Environment, Ghent (Belgium), 29- 30 April 2010, 6p.

De Coensel, B., Can, A., Botteldooren, D., 2012. Effect of traffic signal coordination on noise and air pollutant emissions. Environmental Modelling and Software 35, 74-83.

EC 2002, Directive 2002/49/EC of the European Parliament and of the Council of 25 June 2002, relating to the assessment and management of environmental noise. Official Journal of the European Communities 2002.

El Aarbaoui, T., Méline, J., Brondeel, R., Chaix B., 2017. Short-term association between personal exposure to noise and heart rate variability: The RECORD MultiSensor Study. Environ Pollut, 231(Pt 1), 703-711.

Estevez-Mauriz, L., Forssen, J., 2018. Dynamic traffic noise assessment tool: A comparative study between a roundabout and a signalised intersection. Applied Acoustics 130, 71-86.

European Environmental Agency (EEA), 2014. Good practice guide on quiet areas. EEA Technical report $n^{\circ} 4$, ISSN 1725-2237, 58p. 
Gerike, R., Becker, T., Friedemann, J., Hülsmann, F., Heidegger, F., 2012. Mapping external noise costs to the transport users conceptual issues and empirical results. In Proceedings of the Euronoise 9th European conference on noise control, Prague, 2012, pp. $10-13$.

Gille, L.-A., Marquis-Favre, C., Klein, A., 2016. Noise annoyance due to urban road traffic with powered-two-wheelers: quiet periods, order and number of vehicles. Acta Acustica united with Acustica 102, 474 - 487.

Gulliver, J., Morley, D., Vienneau, D., Fabbri, F., Bell, M., Goodman, P., Beevers, S., Dajnak, D., Kelly, F.J., Fecht, D., 2015. Development of an open-source road traffic noise model for exposure assessment, Environmental Modelling \& Software 74, 183-193.

Gurram, S., Stuart, A.L., Pinjari, A.R., 2019. Agent-based modeling to estimate exposures to urban air pollution from transportation: Exposure disparities and impacts of highresolution data. Computers, Environment and Urban Systems 75, 22-34.

Havard, S., Reich, B.J., Bean, K., Chaix, B., 2011. Social inequalities in residential exposure to road traffic noise: an environmental justice analysis based on the RECORD Cohort Study. Occup Environ Med. 68(5), 366-374.

Heltimo, J., Niitymaki, J., Björk, E., 2003. A microlevel method for road traffic noise prediction. In: Advances in transport 8, urban transport VIII: urban transport and the environment in the 21st century. ISBN 1-85312-905-4.

Hörl, S., and Balac, M., 2020. Open data travel demand synthesis for agent-based transport simulation: A case study for Paris and Île-de-France. Preprint submitted to ETH Research Collection Nov 2020, doi: 10.3929/ethz-b-000412979

Horni, A., Nagel, K., Axhausen, K.W., 2016. Introducing matsim. In: Horni, A., Nagel, K., Axhausen, K.W., editors. The Multi-Agent Transport Simulation MATSim. London: Ubiquity Press, p. 3-8.

Houot, H., Antoni, J.P., Pujol, S., Lamiral, M., Mauny, F., 2015. Les mobilites urbaines et leur impact sur l'exposition au bruit : simulation de scenarios prospectifs appliques à la 
ville de Besancon [Urban mobilities and their impact on noise exposure: simulation of prospective scenarios applied to the city of Besancon]. Transports urbains, G.E.T.U.M. (Groupement pour l'etude des transports urbains modernes), 2015, 16-20.

Kaddoura, I., Kröger L., Nagel K., 2017. An activity-based and dynamic approach to calculate road traffic noise damages. Transportation Research Part D: Transport and Environment 54, 335-347.

Kaddoura, I., 2018. Simultaneous internalization of traffic congestion and noise exposure costs Transportation 45, 1579-1600.

Kephalopoulos, S., Paviotti, M., Anfosso-Lédée, F., 2012. Common Noise Assessment Methods in Europe (CNOSSOS-EU). JRC Reference report 2012.

Kephalopoulos, S., Paviotti, M., and Anfosso-Lédée, F., 2012. Common noise assessment methods in Europe (CNOSSOS-EU) (PUBLICATIONS OFFICE OF THE EUROPEAN UNION), p. 180 p, https: //hal.archives-ouvertes.fr/hal-00985998.

Kuehnel, N., Kaddoura, I., Moeckel, R. 2019. Noise Shielding in an Agent-Based Transport Model Using Volunteered Geographic Data. The 8th International Workshop on Agent-based Mobility, Traffic and Transportation Models, April 29 - May 2, 2019, Leuven, Belgium.

Kuehnel, N., Moeckel R., 2020. Impact of simulation-based traffic noise on rent prices. Transportation Research Part D 78, doi: 10.1016/j.trd.2019.11.020.

Lera-López, F., Faulin, J., Sánchez, M., 2013. Willingness to pay to reduce environmental impacts from road transportation: a case study from the Spanish Pyrenees. Journal of Applied Operational Research 5(4), 135-152.

Lu, M., Schmitz, O., Vaartjes, I., Karssenberg, D., 2019. Activity-based air pollution exposure assessment: Differences between homemakers and cycling commuters. Health \& Place 60, doi:10.1016/j.healthplace.2019.102233.

Luo, W.L., Cai, M., Li, F., Liu, J.K., 2012. Dynamic modeling of road traffic noise around buildings in an urban area. Noise Control Engineering Journal 60(4), 353-362. 
Miedema, H.M., Vos, H., 1998. Exposure-response relationships for transportation noise. Journal of Acoustical Society of America 104, 3432-3445.

Quintero, G., Aumond, P., Can, A., Balastegui, A, Romeu, J., 2019. Statistical requirements for noise mapping based on mobile measurements using bikes. Appl. Acoust. 156, 271-278.

Tang, U.W., Wang, Z.S., 2007. Influences of urban forms on traffic-induced noise and air pollution: Results from a modelling system. Environmental Modelling \& Software 22, 17501764.

Ramacher, M.O.P., Karl, M., 2020. Integrating Modes of Transport in a Dynamic Modelling Approach to Evaluate Population Exposure to Ambient NO2 and PM2.5 Pollution in Urban Areas. Int. J.Environ. Res. Public Health 17, 2099.

Reed, S.E., Boggs, J.L., Mann, J.P., 2012. A GIS tool for modeling anthropogenic noise propagation in natural ecosystems. Environmental Modelling \& Software 37, 1-5.

Rey Gozalo, G., Barrigon Morillas, J.M., 2017. Perceptions and effects of the acoustic environment in quiet residential areas. The Journal of the Acoustical Society of America 141(4), 2418-2429.

Thacher, J.D., Poulsen, A.H., Raaschou-Nielsen, O., Jensen, A., Hillig, K., Roswall, N., Hvidtfeldt, U., Jensen, S.S., Levin, G., Valencia, V.H., Sørensen. M., 2020. High-resolution assessment of road traffic noise exposure in Denmark. Environmental Research 182, doi: 10.1016/j.envres.2019.109051.

Van Kamp, I., Simon, S., Notley, H., Baliatsas, C., Van Kempen, E., 2020. Evidence Relating to Environmental Noise Exposure and Annoyance, Sleep Disturbance, CardioVascular and Metabolic Health Outcomes in the Context of IGCB (N): A Scoping Review of New Evidence. Int. J. Environ. Res. Public Health, 17(9), 3016, https://doi.org/10.3390/ijerph17093016

Vienneau, D., Eze, I.C., Probst-Hensch, N., Röösli, M., 2019. Association between Transportation Noise and Cardio-Metabolic Diseases: An Update of the WHO Meta-Analysis; ICA Proceedings: Aachen, Germany, 2019. 
World Health Organisation (WHO), 2011. Burden of disease from environmental noise. Quantification of healthy life years lost in Europe. ISBN: 978928900229 5, 126p.

World Health Organisation (WHO), 2018. Environmental Noise Guidelines for the European Region. 181p.

Zhuge, C., Shao, C., Wang, S., Hu, Y., 2019. Sensitivity analysis of integrated activitybased model: using MATSim as an example. Transportation Letters 11(2), 93-103.

Zwick, F., Kuehnel, N., Moeckel, R., Axhausen, K.W. 2021. Agent-based simulation of city-wide autonomous ride-pooling and the impact on traffic noise, Transportation Research Part D 90 (2021) 102673 